\title{
ESTUDOS BIOECOLÓGICOS SOBRE Tradescantia fluminensis (Commelinaceae) E SEUS INIMIGOS NATURAIS ASSOCIADOS, NO PARANÁ
}

\author{
José Henrique Pedrosa-Macedo*, Josicler Klug Weigert**, Luiz Alessandro Scapini**, \\ Daiane Niederhartmann**, Dener Rocha Bebiano**, Simon Fowler***, Nicholas Waipara*** \\ *Eng. Florestal, Dr., Depto. de Ciências Florestais, UFPR - johpema@netpar.com.br \\ **Acadêmicos de Eng. Florestal, UFPR - 1ncbp@ufpr.br \\ ***Dr., Manaaki Whenua Landcare Research, Nova Zelândia - fowlers@landcareresearch.nz \\ Recebido para publicação: 14/09/2005 - Aceito para publicação: 31/10/2006
}

\begin{abstract}
Resumo
Tradescantia fluminensis (Commelinaceae) é uma planta invasora e supressora da regeneração natural das florestas da Nova Zelândia. Este trabalho realizou uma busca preliminar de seus inimigos naturais e estudos bioecológicos sobre a planta no estado do Paraná, em particular na Região Metropolitana de Curitiba (RMC), onde a espécie T. fluminensis é de ocorrência natural ao lado de outras da mesma família. O material vegetal e o material entomológico foram coletados em quatro municípios da RMC e foram avaliados, analisados e feitos estudos preliminares em laboratório e dentro de casa de vegetação do Setor de Ciências Agrárias da Universidade Federal do Paraná em Curitiba. Foram conduzidos estudos sobre crescimento de raízes e biomassa da planta. Durante os períodos vegetativos de 2003/4 e 2004/5, foram coletadas 43 espécies de insetos associados à planta. Cinco espécies demonstraram potencial para serem candidatas ao controle biológico de $T$. fluminensis: quatro espécies da família Chrysomelidae e uma espécie de Thrips.

Palavras-chave: Controle biológico; inimigos naturais; planta invasora.
\end{abstract}

\begin{abstract}
Bioecological studies on Tradescantia fluminensis (Commelinaceae) and its natural enemies, in Parana State. Tradescantia fluminensis (Commelinaceae) is an invasive and suppressing plant of the natural regeneration of native forests in New Zeeland. This work conducted a preliminary search of the natural enemies and bioecological studies on the plant in Paraná State, particularly at the Metropolitan Region of Curitiba (RMC), where this species has natural occurrence with others plants of the same family. The vegetal and entomological materials were collected in four Districts of the RMC and were evaluated, analyzed and conducted preliminary studies in laboratory and inside Green House - Agrarian Science Division of the Federal University of Paraná in Curitiba. Studies were conducted on root growing and biomass of the plant. During the period 2003/4 and 2004/5 were colleted 43 species of insects associated to this plant. Five candidates' species demonstrated potential value for Biological Control of T. fluminensis; four species of the family Chrysomelidae and one species of Thrips.

Keywords: Biological control; invasive plant; and natural enemies.
\end{abstract}

\section{INTRODUÇ̃̃O}

O homem, ao cultivar plantas, aprendeu a transportá-las para lugares diferentes de sua origem. No início as plantas serviam para sua alimentação e seus instrumentos rudimentares. À medida que passou a depender delas economicamente, incluindo o tratamento de doenças, jardinagem e arborização de vias públicas, o transporte de plantas passou a ser rotina na vida humana. O fato de transportá-las ganhou velocidade com os meios modernos de transporte. Plantas são transportadas de um continente para outro em pouco tempo, nos quais elas se adaptam e em certos casos tornam-se invasoras e indesejáveis para a economia e o meio ambiente. Esse é o caso da espécie Tradescantia fluminensis Vell. (Commelinaceae) nativa do Brasil meridional e com ampla distribuição em áreas antropizadas. Na Nova Zelândia (TOFT et al., 2001) e na Flórida, Estados Unidos (LANGELAND; BURKS, 1998), onde o 
clima lhe é favorável, é uma invasora economicamente importante. O seu crescimento é vigoroso e forma uma cobertura em forma de "tapete" no sub-bosque das florestas nativas. Com isso, tornou-se um fator supressor e limitante da regeneração natural de florestas. Na Universidade Federal do Paraná, durante o período de dois anos e meio, 2003 a 2005, dentro de um programa cooperativo com a Nova Zelândia, foram realizados levantamentos de campo e estudos bioecológicos preliminares sobre a planta e seus inimigos naturais. Os estudos abrangeram áreas do Primeiro e do Segundo Planalto Paranaense. As informações obtidas servirão como subsídios para o prosseguimento das pesquisas e das futuras introduções de inimigos naturais candidatos ao controle biológico na Nova Zelândia.

Nesta fase inicial do programa de pesquisa, realizou-se como meta o levantamento sobre a ocorrência de $T$. fluminensis, o reconhecimento de inimigos naturais associados à planta, estudos bioecológicos e seleção de candidatos ao controle biológico, incluindo testes de especificidade visando à futura exportação para Nova Zelândia.

\section{MATERIAL E MÉTODOS}

O estudo foi realizado em fragmentos florestais na Região Metropolitana de Curitiba, compreendida entre os meridianos $48^{\circ}-49^{\circ} \mathrm{W}$ e paralelos $25^{\circ}-26^{\circ} \mathrm{S}$. Essa região, de acordo com Carpanezzi et al. (1986), é caracterizada por altitudes entre 650 e 1100 m, e pela Floresta Ombrófila Mista com Araucaria angustifolia (Bertol.) Kuntze (Araucariaceae) e campos submontanos, onde existem apenas fragmentos florestais, os quais são descontínuos e entremeados por horticultura e agricultura de pequeno porte. Nesse ecossistema, concentrou-se e deu-se ênfase aos estudos. No litoral paranaense, foram visitados os municípios de Morretes $\left(25^{\circ} 28^{`} \mathrm{~S}, 48^{\circ} 50^{\prime} \mathrm{W}\right.$ - altitude 5-10 m), Antonina $\left(25^{\circ} 25^{`} \mathrm{~S}\right.$, $48^{\circ} 42^{`} \mathrm{~W}$ - altitude $0 \mathrm{~m}$ ) e Paranaguá $\left(25^{\circ} 31^{`} \mathrm{~S}, 48^{\circ} 30^{\prime} \mathrm{W}\right.$ - altitude $0 \mathrm{~m}$ ), em busca de $T$. fluminensis.

O material vegetal de estudo foram ramos, folhas, entrenós e raízes de T. fluminensis. Foram coletados insetos associados à planta que compuseram o material utilizado nos estudos. Para dimensionar a biomassa por $\mathrm{m}^{2}$, foram tomadas amostras silvestres (raízes, ramos e folhas), cujo material foi homogeneizado através da retirada de impurezas, pesado em balança de braço (Record - $1610 \mathrm{~g}$ ) e secado em estufa (FANEM) a $60{ }^{\circ} \mathrm{C}$ durante 48 horas. Em seguida realizou-se a pesagem final.

Para dimensionar a biomassa do entrenó (com e sem folha), tomaram-se amostras silvestres aleatórias $(n=20)$, sendo dez caules com folhas $(n=10)$, dos quais foram retiradas as folhas, e dez caules sem folhas $(\mathrm{n}=10)$. Fez-se uma pesagem de matéria fresca em balança analítica (BEL - Mod. Mrk 210A). Realizou-se a secagem a $65^{\circ} \mathrm{C}$ durante 24 horas e o material foi repesado para se obter a biomassa.

Foram tomadas aleatoriamente 30 folhas e destas recortadas áreas foliares circulares de $2 \mathrm{~cm}^{2}$ cada, para dimensionar a biomassa e verificar a sua densidade. Esse material foi pesado verde e levado à secagem em estufa (Odontobras Mod. E.L1.3) a $60{ }^{\circ} \mathrm{C}$ durante 24 horas e repesado para se obter a biomassa.

Para dimensionar o murchamento natural de folhas, em recipientes, foram tomadas aleatoriamente amostras de folhas cultivadas $(\mathrm{n}=30)$, isoladas individualmente em potes plásticos de $50 \mathrm{ml}$ e tampados para conservar o mesmo ambiente em que foram submetidos os espécimes no experimento de consumo de massa verde. Durante o experimento (192 horas), a temperatura ambiente variou entre 17,1 e $28,6^{\circ} \mathrm{C}$ e a umidade relativa entre 54 e $99 \%$. As amostras foram pesadas (BEL-Mod. Mrk 210A) a cada 24 horas.

Para determinar a relação bainha/entrenó, foram dimensionadas amostras $(\mathrm{n}=80)$ nas quais havia 80 bainhas e 80 entrenós, constituídas de T. fluminensis $(\mathrm{n}=60)$ e de Commelina diffusa Burn. $(n=20)$, cujo objetivo foi encontrar um coeficiente que mostre a diferença entre as duas espécies desses gêneros distintos.

Para o teste de propagação vegetativa, foram tomadas aleatoriamente nove amostras (ramos) $(\mathrm{n}=9)$, e de cada amostra foram cortados oito entrenós, sendo quatro na parte superior do caule com folhas $(n=4)$ e quatro na parte inferior sem folhas $(n=4)$, os quais foram dimensionados: comprimento $(\mathrm{cm})$, diâmetro $(\mathrm{mm})$ e peso $(\mathrm{g})$. Em seguida, o material foi colocado em placa de Petri para o acompanhamento diário do enraizamento em laboratório.

O material entomológico foi coletado em excursões de campo nas seguintes localidades: Mananciais da Serra - Piraquara $\left(25^{\circ} 30^{\prime} \mathrm{S}-48^{\circ} 45^{\prime} \mathrm{W}\right)$; Juruqui - Almirante Tamandaré $\left(25^{\circ} 16^{\prime} \mathrm{S}-\right.$

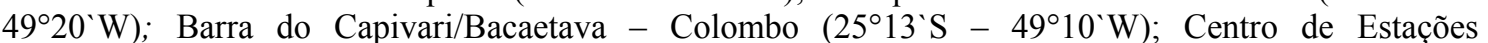
Experimentais do Canguiri (CEEC) - Pinhais $\left(25^{\circ} 24^{\prime} \mathrm{S}-49^{\circ} 15^{`} \mathrm{~W}\right)$ e Arboreto Juvevê-UFPR - Curitiba 
$\left(25^{\circ} 21^{\prime} \mathrm{S}-49^{\circ} 14 \mathrm{~W}\right)$. Foi realizada uma excursão ao Parque Estadual de Vila Velha $\left(25^{\circ} 15^{\prime} \mathrm{S}-\right.$ $50^{\circ} 00^{\prime} \mathrm{W}$ ), em Ponta Grossa (Segundo Planalto Paranaense).

Os insetos associados à T. fluminensis foram coletados e/ou capturados manualmente, mortos, identificados e alfinetados para compor uma coleção.

Para determinar o consumo de material fresco (verde), foram tomados nove exemplares silvestres de uma espécie da família Chrysomelidae $(n=9)$, confinados isoladamente em potes de plástico de $50 \mathrm{ml}$ com identificação individual. O procedimento adotado obedeceu ao seguinte critério: diariamente as folhas, previamente pesadas na balança analítica (BEL - Mod. Mark 210A), eram fornecidas aos espécimes e repesadas no dia seguinte, depois de danificadas. Também foram utilizados os restos das folhas para determinação da biomassa das sobras de folhas. A diferença entre o peso (mg) da folha íntegra, fornecida no dia anterior, e a danificada após 24 horas, forneceu o consumo diário de massa verde. Durante o período de execução do experimento, a temperatura mínima variou de 15,8 a $17,1^{\circ} \mathrm{C}$, e a máxima de 19,5 a $28,6^{\circ} \mathrm{C}$. A umidade relativa variou de 60 a $99 \%$. Os dados foram submetidos à análise de variância (ANOVA) e as médias comparadas pelo teste de Tukey, $\mathrm{p} \leq 0,05$.

Uma espécie da família Chrysomelidae foi submetida aos testes de preferência com e sem escolha usando dois ambientes: a) placa de Petri e b) gaiola de vidro $(15 \times 15 \times 30 \mathrm{~cm})$ com tampa de nylon. Foram usadas nos testes as seguintes plantas: Solanum melongena L. (Solanaceae) - berinjela; Capsicum sp. (Solanaceae) - pimenta; Solanum gilo Raddi (Solanaceae) - jiló; e Phaseolus vulgaris L. (Leguminosae) - feijão; todas cultivadas pelo LNCBP.

Foram adquiridas no mercado outras plantas para testes em placa de Petri, conforme segue: Capsicum annuum L. (Solanaceae) - pimentão; Lactura sativa L. (Asteraceae) - alface; Nasturtium officinale L. (Brassicaceae) - agrião; e Spinacia oleracea L. (Amaranthaceae) - espinafre (Tabelas 1 e 2).

Foram registrados aspectos comportamentais de duas espécies em campo e laboratório, entre eles aspectos da atividade sazonal, o ritmo diário, os tipos de danos causados pelo adulto e pela larva, o acasalamento, a postura e o número de ovos.

Tabela 1. Teste de preferência com T. fluminensis + material vegetal com escolha e sem escolha com uma espécie de crisomelídeo, conduzido em gaiola de vidro com planta em vaso $(24 \times 23 \mathrm{~cm})$.

Table 1. Preference test with $T$. fluminensis + choice vegetable material and no choice with chrysomelid species. Conducted in glass cage with plant in pot $(24 \times 23 \mathrm{~cm})$.

\begin{tabular}{lcc}
\hline Vaso & Plantas testadas & Teste \\
\hline 01 & Tradescantia + Lycopersicum esculentum & Com escolha \\
02 & Lycopersicum esculentum & Sem escolha \\
03 & Tradescantia + Solanum melogena & Com escolha \\
04 & Solanum melogena & Sem escolha \\
05 & Tradescantia + Capsicum sp. & Com escolha \\
06 & Capsicum sp. & Sem escolha \\
07 & Tradescantia + Solanum gilo & Com escolha \\
08 & Solanum gilo & Sem escolha \\
\hline
\end{tabular}

Tabela 2. Teste de preferência com T. fluminensis + material vegetal com escolha e sem escolha com uma espécie de crisomelídeo, conduzido em placa de Petri.

Table 2. Preference test with $T$. fluminensis + choice vegetable material and no choice with chrysomelid species, conducted in glass cage in plant in Petri disches.

\begin{tabular}{lcc}
\hline Vaso & Plantas testadas & Teste \\
\hline 01 & Tradescantia + Lactura sativa & Com escolha \\
02 & Lactura sativa & Sem escolha \\
03 & Tradescantia + Spinacia oleracea & Com escolha \\
04 & Spinacia oleracea & Sem escolha \\
05 & Tradescantia + Nasturtium officinale & Com escolha \\
06 & Nasturtium officinale & Sem escolha \\
07 & Tradescantia + Phaseolus vulgaris & Com escolha \\
08 & Phaseolus vulgaris & Sem escolha \\
09 & Tradescantia + Tradescantia zebrina & Com escolha \\
10 & Tradescantia zebrina & Sem escolha \\
\hline
\end{tabular}




\section{RESULTADOS E DISCUSSÃO}

Em todas as localidades da Região Metropolitana de Curitiba, a espécie T. fluminensis ocorre naturalmente em sítios úmidos ou margens alagadiças de rios e riachos, raramente em sítios secos do interior de fragmentos florestais. Nos sítios cobertos por vegetação arbórea, a espécie T. fluminensis forma densa "cobertura verde" no subosque de fragmentos florestais. Estes são sítios antropofizados entre as coordenadas geográficas $25^{\circ} 10^{\prime}-25^{\circ} 45^{\prime} \mathrm{S}$ e $49^{\circ} 00^{\prime}-49^{\circ} 30^{\prime} \mathrm{W}$ - altitude de $950-1000 \mathrm{~m}$. As coletas abrangeram os municípios de Curitiba, Pinhais, Almirante Tamandaré, Campo Magro, Colombo, Quatro Barras e Bocaiúva do Sul. No Segundo Planalto Paranaense (altitude de 917 m), Parque Estadual de Vila Velha, a planta encontra-se vegetando junto ao solo de arenito, porém em nichos sombreados e úmidos. No litoral paranaense, T. fluminensis não foi encontrada.

Essa planta brasileira é uma erva decumbente de até $20 \mathrm{~cm}$ de altura, caule verde ou arroxeado, sulcado, dividido em nós glabros. Suas folhas são ovadas e de ápice acuminado, alternas, glabras, subcarnosas, curvinérveas, bainha fechada com anel de pêlos apicais. Não há registros na literatura sobre a sua frutificação. Sua propagação é por estolho, que é o seu maior dispersor. Sua flor trímera é branca e terminal. Apesar de florescer em quase todas as estações do ano, não produz semente. No Paraná, além da Região Metropolitana de Curitiba, sua presença é registrada no Parque Estadual de Vila Velha e no Terceiro Planalto Paranaense (TAKEDA; FARAGO, 2001). Mendonça et al. (1998), numa relação de Commelinaceae, citam-na como sendo uma das plantas "fanerógamas do bioma cerrado brasileiro", cujo hábitat são as matas de galeria.

$\mathrm{Na}$ Nova Zelândia, T. fluminensis tem impedido e suprimido a regeneração de florestas. Nesse país, ela atinge até $80 \mathrm{~cm}$ de altura, o que não acontece na RMC, onde a altura atinge até $40 \mathrm{~cm}$.

Commelina diffusa é comum na cidade de Curitiba e se confunde com T. fluminensis. A ocorrência de $C$. diffusa foi registrada, também, em todos os municípios da RMC, incluindo aqueles do litoral paranaense, onde T. fluminensis não ocorre. Quando não há floração, torna-se difícil distinguir $C$. diffusa de T. fluminensis. Por isso, procurou-se estabelecer o "coeficiente bainha/entrenó", sendo este 0,30 para C. diffusa e 0,13 para T. fluminensis (Figura 1). As espécies Tradescantia zebrina Heynh. e Tradescantia pallida Rose são encontradas com freqüência na RMC e são usadas como ornamentais em jardins e praças, confirmando a indicação de Blossfeld (1965), porém não são invasoras, como é o caso de T. fluminensis.

O coeficiente médio (bainha/entrenó) demonstrou ser 2,3 vezes maior para C. diffusa $(0,30)$ quando comparado com $T$. fluminensis $(0,13)$, conforme tabela 3 e figura 1.

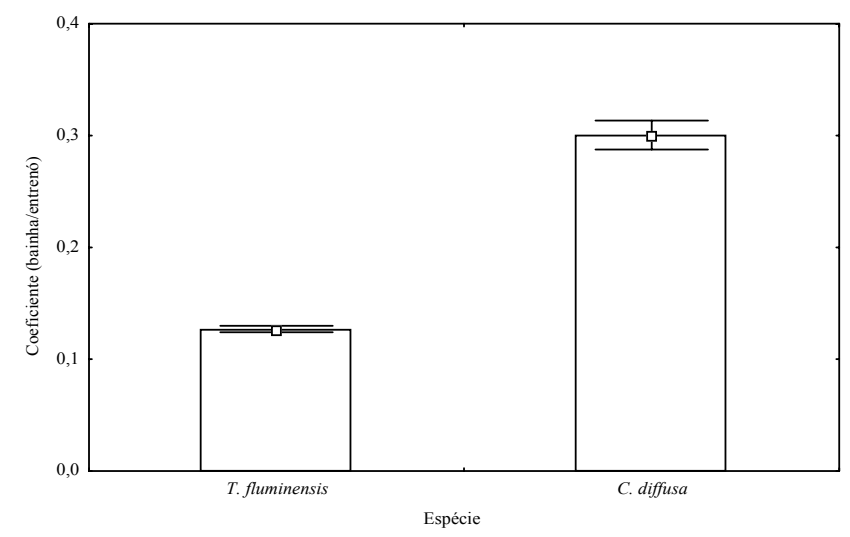

Figura 1. Coeficiente (bainha/entrenó) de T. fluminensis $(0,13)$ e C. diffusa $(0,30)$.

Figure 1. Coefficient (node/internodes) of T. fluminensis $(0,13)$ and C. diffusa $(0,30)$.

Ramos sem folhas produzem cerca de três vezes mais raízes do que ramos com folhas e em menor tempo (Tabela 4 e Figura 2). 
Tabela 3. Análise estatística do coeficiente (bainha/entrenó) de $T$. fluminensis e $C$. diffusa.

Table 3. Statistical analyses of the coefficient (node/internodes) of T. fluminensis and C. diffusa.

\begin{tabular}{lcccccc}
\hline Planta & Média & Std.Dv. & N & T & df & P \\
Coeficiente $T$. fluminensis & 0,127 & 0,023 & 60 & \multirow{2}{*}{19,95} & \multirow{2}{*}{78} & \multirow{2}{*}{0,000000} \\
Coeficiente C. diffusa & 0,301 & 0,054 & 20 & & \\
\hline
\end{tabular}

Tabela 4. Análise estatística (teste t) de produção de raízes em ramos com folhas e sem folhas.

Table 4. Statistical analyses ( $\mathrm{t}$ test) of roots production in stems with leaves and without leaves.

\begin{tabular}{lcccccccc}
\hline & N & Média & IC (95\%) & Min & Max & Var & DP & EP \\
\hline Com folhas & 9 & 2,778 & 4,09676 & 1 & 6 & 2,944 & 1,716 & 0,572 \\
Sem folhas & 9 & 8,556 & 10,5933 & 5 & 12 & 7,028 & 2,651 & 0,884 \\
\hline
\end{tabular}

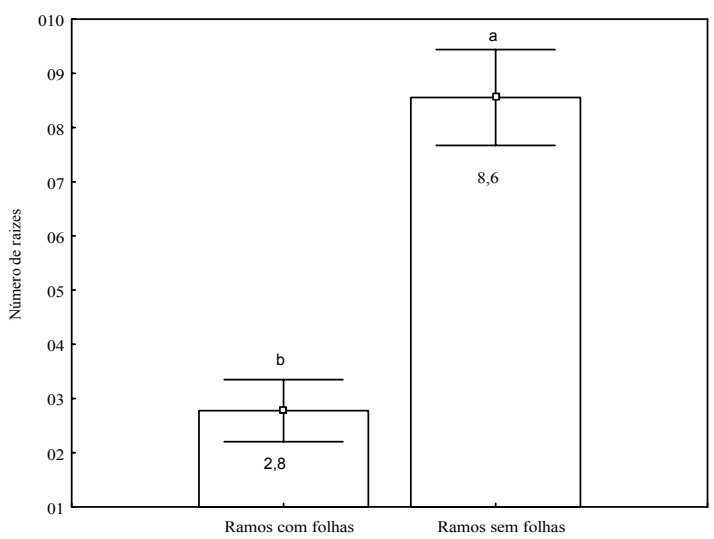

Figura 2. Ramos sem folhas $(8,6)$ produzem, em média, três vezes mais raízes do que os com folhas $(2,8)$.

Figure 2. Stems without leaves $(8,6)$ produced on average tree times more roots than stems with leaves $(2,8)$.

A biomassa por metro quadrado $\left(\mathrm{m}^{2}\right)$ de $T$. fluminensis variou de acordo com a densidade da população e em cada município do Primeiro Planalto Paranaense, onde foram tomadas as amostras (Tabela 5 e Figura 3). A secagem do material exigiu um período de $48 \mathrm{~h}$ a $60{ }^{\circ} \mathrm{C}$.

Tabela 5. Análise estatística da biomassa $\left(\mathrm{m}^{2}\right)$ de $T$. fluminensis no Primeiro Planalto do estado do Paraná.

Table 5. Statistical analyses of T. fluminensis biomass $\left(\mathrm{m}^{2}\right)$ in the First Plateau of Paraná State.

\begin{tabular}{lcccc}
\hline & Origem & N & Média \pm DP & Var. \\
\hline Peso verde & Juvevê Arboretum $(*)$ & 2 & $779,20 \pm 340,40$ & 115872,98 \\
& Juruqui (Primavera) $(* *)$ & 3 & $1665,17 \pm 486,07$ & 236265,90 \\
& Bacaetava $(* * *)$ & 3 & $1686,00 \pm 496,69$ & 246700,00 \\
\hline Biomassa & Juvevê Arboretum $(*)$ & 2 & $63,55 \pm 24,68$ & 609,01 \\
& Juruqui (Primavera) $(* *)$ & 3 & $135,43 \pm 53,14$ & 2823,88 \\
& Bacaetava $(* * *)$ & 3 & $211,21 \pm 73,83$ & 5450,58 \\
\hline
\end{tabular}

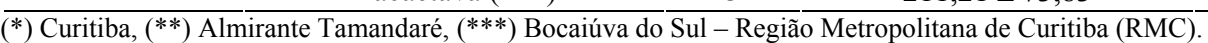

Ramos com folhas têm $27 \%$ de biomassa a mais do que ramos sem folhas. Em média, a biomassa de ramos com folhas pesa $559 \pm 12 \mu \mathrm{g}$, enquanto que a biomassa média de ramos sem folhas (entrenó) é de $408 \pm 11 \mu \mathrm{g}$ (Tabela 5 e Figura 2). No caso da secagem de ramos sem folhas (entrenó), foram suficientes $24 \mathrm{~h}$ à temperatura de $65^{\circ} \mathrm{C}$.

Os resultados do experimento de biomassa de área foliar em $\mathrm{cm}^{2}(\mathrm{n}=30)$ demonstraram que existe flutuação de densidade em cada folha. O peso de material fresco (verde), a água e outros, e a 
biomassa nas folhas de $T$. fluminensis oscilam conforme mostra a figura 4a. A média por centímetro quadrado do peso verde é de $436,41 \mu \mathrm{g} / \mathrm{cm}^{2}$, enquanto que a média de água e outros é de $397,88 \mu \mathrm{g} / \mathrm{cm}^{2}$, e a biomassa é de $38,5 \mu \mathrm{g} / \mathrm{cm}^{2}$ (Figura $4 \mathrm{~b}$ ). Nesta figura os parâmetros estão representados em dobro. Por conveniência operacional, o experimento foi conduzido com uma área foliar de $2 \mathrm{~cm}^{2}$ (Figuras $4 \mathrm{a}$ e $4 \mathrm{~b}$ ).

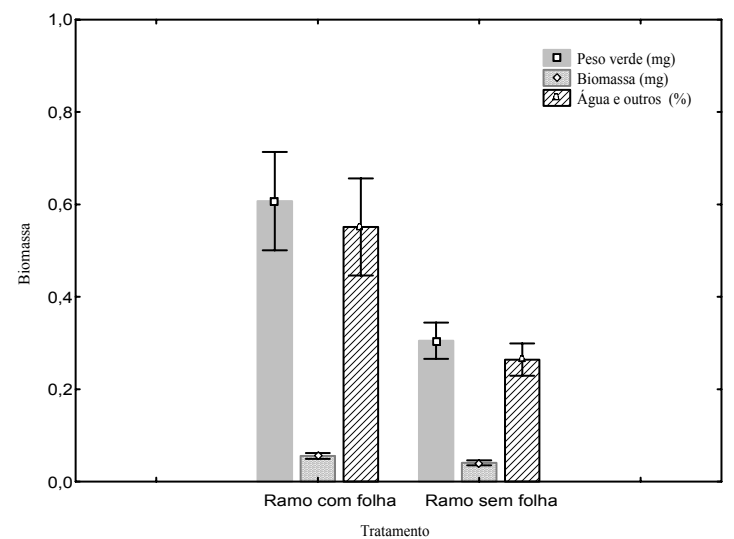

Figura 3. A biomassa média no ramo com folha e do ramo sem folha.

Figure 3. Biomass average in the stem with leaf and without leaf.

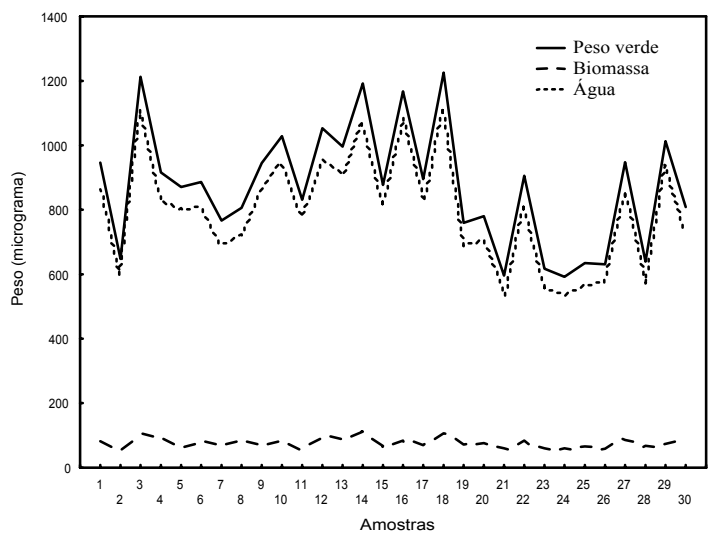

(a)

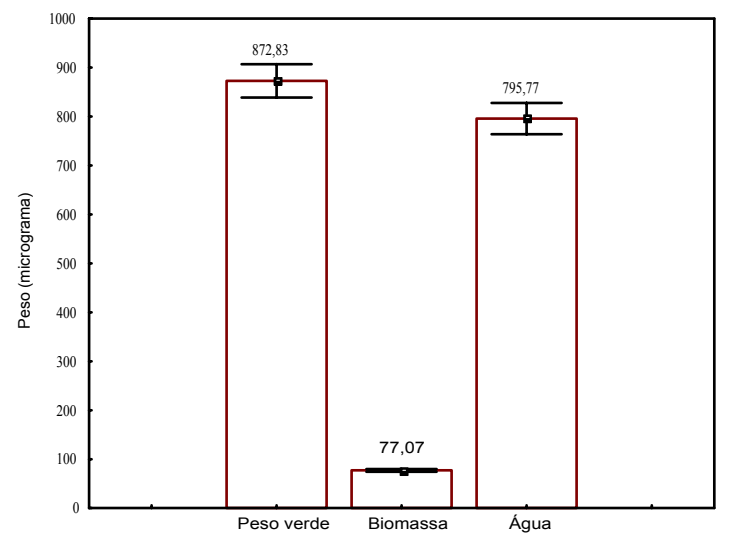

(b)

Figura 4. Flutuação do peso verde, da água e outros e da biomassa de folhas de T. fluminensis $(\mathrm{n}=30)$. (a) Componentes médios de $2 \mathrm{~cm}^{2}$ da folha de $T$. fluminensis, biomassa $\left(77,07 \mu \mathrm{g} / \mathrm{cm}^{2}\right)$, água e outros líquidos $\left(795,77 \mu \mathrm{g} / \mathrm{cm}^{2}\right)$; (b) Componentes médios de $2 \mathrm{~cm}^{2}$ da folha de T. fluminensis, biomassa $\left(77,07 \mu \mathrm{g} / \mathrm{cm}^{2}\right)$, água e outros líquidos $\left(795,77 \mu \mathrm{g} / \mathrm{cm}^{2}\right)$.

Figure 4. Average fluctuation of the green weight, water and other liquids, and leaf biomass of $T$. fluminensis $(\mathrm{n}=30)$. (a). Average components per $2 \mathrm{~cm}^{2}$ of $T$. fluminensis leaf, biomass $(77,07$ $\left.\mu \mathrm{g} / \mathrm{cm}^{2}\right)$, water and other liquids $\left(795,77 \mu \mathrm{g} / \mathrm{cm}^{2}\right)$. (b) Average components per $2 \mathrm{~cm}^{2}$ of $T$. fluminensis leaf, biomass $\left(77,07 \mu \mathrm{g} / \mathrm{cm}^{2}\right)$, water and other liquids $\left(795,77 \mu \mathrm{g} / \mathrm{cm}^{2}\right)$.

Comparando a biomassa média de folhas íntegras, que foi de 347,6 $\pm 92,6 \mu \mathrm{g}(\mathrm{n}=30)$ (Tabela 6), com a biomassa de folhas danificadas, que foi de $209,8 \pm 55 \mu \mathrm{g}(\mathrm{n}=45)$, durante 24 horas, verifica-se uma diferença de 137,8 $\mu \mathrm{g}$, o que teoricamente correspondeu ao consumo médio de biomassa pelo crisomelídeo durante cinco dias (120 h) (Tabela 6, Figura 5). Deve ser considerado, no entanto, que no período de 24 horas uma folha de $T$. fluminensis perde cerca de $4,7 \%$ de água e outros líquidos pelo murchamento, conforme está demonstrado a seguir. Portanto, esse consumo é aproximado e não o real. 
Tabela 6. Avaliação estatística de peso verde, biomassa e quantidade de água de amostras de $2 \mathrm{~cm}^{2}$ de folhas de T. fluminensis.

Table 6. Statistical analyses of green weight, biomass and amount of water in $2 \mathrm{~cm}^{2}$ leave samples of T. fluminensis.

\begin{tabular}{lcccccccc}
\hline & N & Média & IC (95\%) & Min. & Max. & Var. & DP & EP \\
\hline Peso verde & 30 & 872,83 & 942,98 & 592 & 1225 & 35290,83 & 187,86 & 34,30 \\
Biomassa & 30 & 77,07 & 82,94 & 49 & 112 & 247,24 & 15,72 & 2,87 \\
$\mathrm{H}_{2} \mathrm{O}$ & 30 & 795,77 & 861,02 & 532 & 1118 & 30539,63 & 174,76 & 31,91 \\
\hline
\end{tabular}

Tabela 7. Avaliação estatística da biomassa de folha íntegra de T. fluminensis e folha danificada por $B$. cinctipennis.

Table 7. Statistical analyses of the whole leaf biomass of $T$. fluminensis and damaged leaf by $B$. cinctipennis.

\begin{tabular}{lccccccc}
\hline & N & Média & Min. & Max. & Var. & DP & EP \\
\hline Folha íntegra (biomassa) & 30 & 347,57 & 175 & 525 & 8580,12 & 92,63 & 16,91 \\
Folha danificada (biomassa) & 45 & 209,80 & 37 & 332 & 3053,44 & 55,26 & 8,24 \\
\hline
\end{tabular}

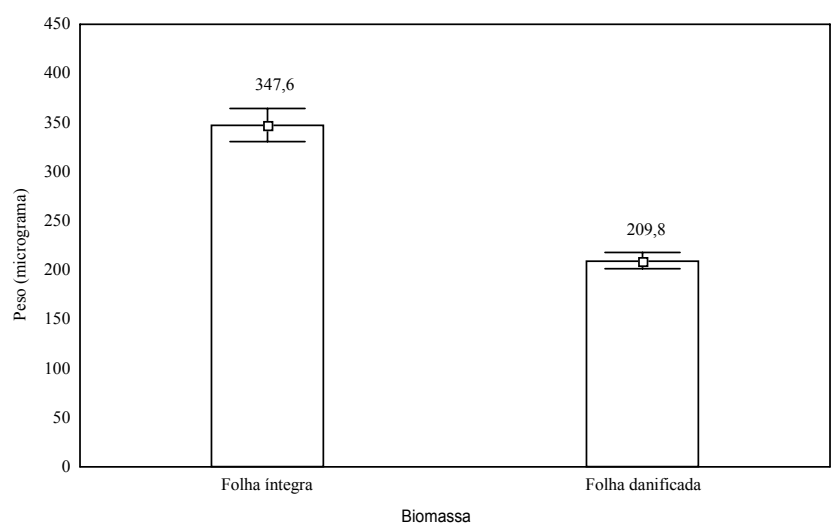

Figura 5. Biomassa de folhas íntegras de T. fluminensis $(\mathrm{n}=30)$ e folhas danificadas $(\mathrm{n}=45)$ por $B$. cinctipennis.

Figure 5. Biomass of the whole leaves of T. fluminensis $(\mathrm{n}=30)$ and of damaged leaves $(\mathrm{n}=45)$ by $B$. cinctipennis.

Por outro lado, o consumo diário de material fresco (verde) pelo crisomelídeo foi de $420 \mu \mathrm{g} / 24 \mathrm{~h}$, ou seja, um consumo médio de $17,5 \mu \mathrm{g} / \mathrm{h}$. Esse parâmetro foi estimado por procedimento indireto.

A evolução do murchamento de folhas $(n=10)$ foi monitorada em teste piloto durante um período de quatro dias $(96 \mathrm{~h}$ ), e registrou-se que há um ritmo regular de perda de água e outros componentes (Tabela 8). Esse fenômeno ficou comprovado em um novo teste, o qual foi conduzido com maior número de folhas $(\mathrm{n}=30)$, durante $192 \mathrm{~h}$ (oito dias). Há regularidade em cada folha, e essa regularidade se manteve constante e decrescente durante o teste (Tabelas 8 e 9, Figuras 6a e 6b). A partir de $120 \mathrm{~h}$ (cinco dias), houve diferença estatística comprovada em relação ao início do experimento.

Foram coletadas 43 espécies de insetos pertencentes a sete ordens. A ordem mais abundante foi Coleóptera, com 23 espécies (53,6\%), sendo que a família Chrysomelidae, com cinco subfamílias (Criocerinae, Alticinae, Cassidinae, Chrysomelinae e Galerucinae), foi a mais freqüente, com 15 espécies. Destas, apenas uma espécie foi identificada: Bruckibrotica cinctipennis Baly (Coleoptera: Chrysomelidae). A espécie de criocerineo (Coleoptera: Chrysomelidae) está em processo de identificação, com suspeita de tratar-se de uma espécie nova. Em seguida, as famílias mais abundantes foram Coccinellidae ( 2 espécies) e Curculionidae ( 2 espécies), seguidas por Carabidae, Ptilodactylidae, Oedemeridae e Elateridae (uma espécie cada). Toft et al. (2001), trabalhando com armadilha de Malaise (Nova Zelândia), coletaram espécies das famílias Chrysomelidae, Curculionidae e Coccinellidae. A ordem Díptera, com sete espécies (16,3\%), vem em segundo lugar em abundância, todas preliminarmente 
identificadas como sendo pertencentes à família Luxaniidae. A ordem Hemiptera apresentou seis espécies $(13,9 \%)$, pertencentes à família Pentatomidae e Cicadelidae, respectivamente. A ordem Hymenoptera vem em seguida, com quatro espécies $(9,3 \%)$, pertencentes à família Braconidae. Por fim, as ordens Lepidoptera, Psocoptera e a Thysanoptera, com uma espécie cada (2,3\%). A espécie da ordem Lepidoptera é da família Noctuidae, cuja maioria das espécies são pragas de sementeiras e viveiros.

Tabela 8. Avaliação estatística do peso de folhas $(\mu \mathrm{g})$ de biomassa de $T$. fluminensis durante cinco dias.

Table 8. Statistical analyses of the leaves weight $(\mu \mathrm{g})$ of $T$. fluminensis and biomass during five days.

\begin{tabular}{lcccccccc}
\hline $\begin{array}{l}\text { Período de } \\
\text { pesagem } \\
\text { (horas) }\end{array}$ & $\begin{array}{c}\text { Número de } \\
\text { amostras } \\
\text { (N) }\end{array}$ & $\begin{array}{c}\text { Perda de peso } \\
\text { verde e biomassa } \\
\text { (média) }\end{array}$ & $\begin{array}{c}\text { IC } \\
\mathbf{( 9 5 \% )}\end{array}$ & Min & Max & Var & DP & EP \\
\hline 0 & 10 & 3339,4 & 3813,50 & 2047 & 4306 & 439236,5 & 662,75 & 209,58 \\
24 & 10 & 2779,2 & 3170,84 & 1733 & 3680 & 299735,5 & 547,48 & 173,13 \\
48 & 10 & 2340,1 & 2698,51 & 1414 & 3181 & 251018,3 & 501,02 & 158,44 \\
72 & 10 & 2120,2 & 2446,45 & 1311 & 2931 & 208001,5 & 456,07 & 144,22 \\
96 & 10 & 458,0 & 541,70 & 263 & 655 & 13691,33 & 117,01 & 37,00 \\
\hline
\end{tabular}

Tabela 9. Avaliação estatística da perda de peso de folhas de T. fluminensis durante 192 horas e da biomassa.

Table: 9. Statistical analyses of the leaves weight loss $(\mu \mathrm{g})$ during 192 hours of $T$. fluminensis and biomass.

\begin{tabular}{lcccccccc}
\hline $\begin{array}{l}\text { Período de } \\
\text { pesagem } \\
\text { (horas) }\end{array}$ & $\begin{array}{c}\text { Número de } \\
\text { amostras } \\
\text { (N) }\end{array}$ & $\begin{array}{c}\text { Perda de peso } \\
\text { verde e biomassa } \\
\text { (média) }\end{array}$ & $\begin{array}{c}\text { IC } \\
\mathbf{( 9 5 \% )}\end{array}$ & Min & Max & Var & DP & EP \\
\hline 0 & 30 & 0,3142 & 0,3440 & 0,1230 & 0,4435 & 0,0064 & 0,0799 & 0,0146 \\
24 & 30 & 0,2992 & 0,3260 & 0,1848 & 0,4282 & 0,0051 & 0,0717 & 0,0131 \\
48 & 30 & 0,2827 & 0,3089 & 0,1711 & 0,4136 & 0,0049 & 0,0702 & 0,0128 \\
72 & 30 & 0,2712 & 0,2970 & 0,1516 & 0,4038 & 0,0048 & 0,0691 & 0,0126 \\
96 & 30 & 0,2632 & 0,2887 & 0,1388 & 0,3979 & 0,0047 & 0,0684 & 0,0125 \\
120 & 30 & 0,2574 & 0,2828 & 0,1319 & 0,3926 & 0,0046 & 0,0680 & 0,0124 \\
144 & 30 & 0,2520 & 0,2772 & 0,1250 & 0,3871 & 0,0046 & 0,0675 & 0,0123 \\
168 & 30 & 0,2458 & 0,2708 & 0,1208 & 0,3787 & 0,0045 & 0,0668 & 0,0122 \\
192 & 30 & 0,2395 & 0,2639 & 0,1149 & 0,3688 & 0,0043 & 0,0655 & 0,0120 \\
Biomassa & 30 & 0,0348 & 0,0382 & 0,0175 & 0,0525 & 0,0001 & 0,0093 & 0,0017 \\
\hline
\end{tabular}

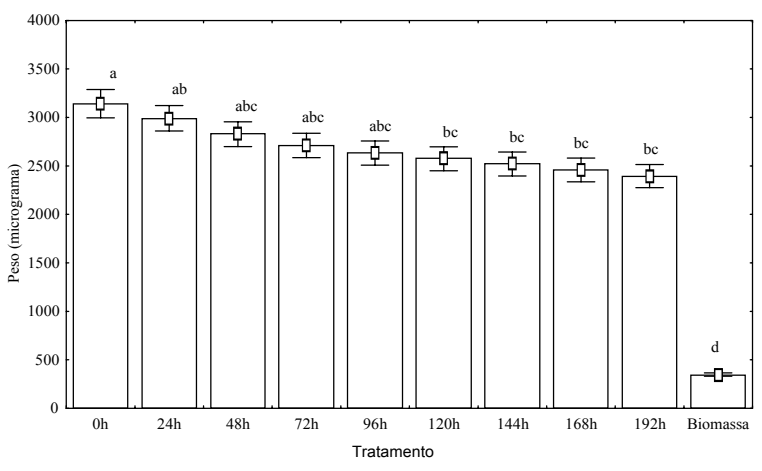

(a)

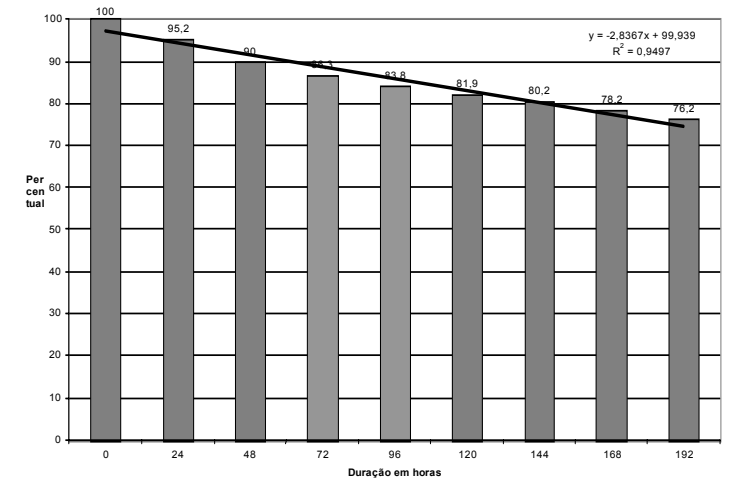

(b)

Figura 6. (a) Evolução da perda de peso de folhas de T. fluminensis durante 192 horas e da biomassa; (b) Avaliação estatística da perda em \% médio de folhas de $T$. fluminensis em potes de $50 \mathrm{ml}$ no laboratório. $\mathrm{Y}=2.8367 \mathrm{x}+99,939 . \mathrm{R}^{2}=0,9497$.

Figure 6. (a) Evolution of the leaves weight loss of T. fluminensis in pot during 192 hours and biomass; (b) Statistical evaluation of the leaves weight loss in \% medium of T. fluminensis in $50 \mathrm{ml}$ pot in the lab. $\mathrm{Y}=2.8367 \mathrm{x}+99,939 . \mathrm{R}^{2}=0,9497$. 
O material entomológico foi encaminhado ao Centro de Identificação de Insetos, Departamento de Zoologia/Entomologia da Universidade Federal do Paraná, para identificação e registro junto à Coleção Jesus Santiago Moure/UFPR.

Uma espécie do gênero Scirtothrips (Thysanoptera:Thripidae) causa danos nos botões apicais e nas folhas de $T$. fluminensis, caracterizados pelo enrolamento parcial de folhas e botões apicais, os quais passam a apresentar pequenas manchas castanhas e necrosadas.

Não foram encontrados parasitóides e nem predadores de adultos e de larvas de $B$. cinctipennis e do besouro crioceríneo. Entretanto, espécimes caem ocasionalmente em teias de aracnídeos e são devorados. Já no caso de Scirtothrips sp. foram encontrados três espécimes de aracnídeos, não identificados, predadores de ninfas.

Os primeiros adultos das espécies $B$. cinctipennis e de crioceríneo aparecem no campo entre a segunda e a terceira semana da primavera, sendo que os espécimes de $B$. cinctipennis são os primeiros a ser encontrados. A atividade do ritmo diário inicia-se por volta das 10:00 h, quando a temperatura atinge 20 a $25^{\circ} \mathrm{C}$. Ao perceberem aproximação, fogem rapidamente em vôo curto. Para capturá-los, é necessário usar um recipiente transparente. Com rede entomológica é difícil capturá-los, pois o vôo ocorre nas proximidades de ramos e folhas de T. fluminensis. As fêmeas dessas duas espécies são mais robustas do que os machos. São encontrados isoladamente sobre as folhas e ramos de T. fluminensis e/ou sobre a vegetação rasteira. No campo, não foi observado o acasalamento. No laboratório, foram registrados acasalamentos com duração de cerca de 10 minutos. A postura é feita sobre folhas e ramos. Foram dissecadas quatro fêmeas de $B$. cinctipennis. A primeira tinha 16 ovos no seu ovário, a segunda tinha 26, sendo 14 ovos maduros, 10 imaturos e dois em formação. Na terceira fêmea, que era menor em relação às anteriores, foram encontrados no seu ovário apenas oito ovos imaturos. No ovário da quarta fêmea foram encontrados 39 ovos, média de 22,25 ovos/fêmea. O ovo $(0,5-1,0 \mathrm{~mm})$ tem cor castanha e forma ovóide, a textura pontuada (células) com minúsculos hexágonos. No período de oito dias, registrou-se a postura de 71 ovos por nove fêmeas (média de 7,9 ovos/fêmea), correspondendo a um ovo/dia. A incubação dura cerca de uma semana. As larvas causam danos nas folhas e nos ramos e seu empupamento é no solo. Observou-se apenas uma geração por ano de B. cinctipennis.

Os testes de preferência alimentar, em placa de Petri e gaiola, demonstraram que $B$. cinctipennis prefere $T$. fluminensis, mas nos testes sem escolha, espinafre (S. oleracea), alface (L. sativa), agrião $(N$. officinalle) e T. zebrina foram atacados (Tabela 10). Outras plantas de importância comercial, como jiló (S. gilo), beringela (S. melongena), pimenta (Capsicum sp.) e feijão (Phaseolus vulgaris L.) não foram atacadas.

Tabela 10.Teste de preferência alimentar de B. cinctipennis (Coleoptera: Chrysomelidae), com chance e sem chance de escolha.

Table 10. Feeding preference test of B. cinctipennis (Coleoptera: Chrysomelidae) with choice and no choice.

\begin{tabular}{|c|c|c|c|c|c|c|}
\hline \multicolumn{3}{|c|}{ Planta hospedeira } & \multicolumn{4}{|c|}{ Ambiente de teste } \\
\hline \multirow{2}{*}{$\begin{array}{l}\text { Nome } \\
\text { comum }\end{array}$} & \multirow{2}{*}{$\begin{array}{c}\text { Nome } \\
\text { científico }\end{array}$} & \multirow[b]{2}{*}{ Família } & \multicolumn{2}{|c|}{ Petri } & \multicolumn{2}{|c|}{ Gaiola } \\
\hline & & & $\begin{array}{c}\text { Sem chance } \\
\text { de escolha }\end{array}$ & $\begin{array}{c}\text { Com chance } \\
\text { de escolha }\end{array}$ & $\begin{array}{c}\text { Sem chance } \\
\text { de escolha }\end{array}$ & $\begin{array}{c}\text { Com chance } \\
\text { de escolha }\end{array}$ \\
\hline Agrião & N. officinalle & Brassicaceae & 0 & 1 & NT & NT \\
\hline Alface & L. sativa & Asteraceae & 1 & 0 & NT & NT \\
\hline Espinafre & S. oleracea & Chenopodiaceae & 1 & 1 & NT & NT \\
\hline T. zebrina & T. zebrina & Commelinaceae & 1 & NT & NT & NT \\
\hline Beringela & S. melongena & Solanaceae & NT & NT & 0 & 0 \\
\hline Feijão & P. vulgaris & Fabaceae & 0 & 0 & NT & NT \\
\hline Jiló & S. gilo & Solanaceae & NT & NT & 0 & 0 \\
\hline Pimenta & Capsicum sp. & Solanaceae & NT & NT & 0 & 0 \\
\hline Tomate & L. esculentum & Solanaceae & NT & NT & 0 & 0 \\
\hline
\end{tabular}

0: não danificado (no damaged); 1: danificado (damaged); NT: não testado (no tested).

A espécie de besouro crioceríneo foi coletada pela primeira vez em 02 de setembro de 2004, no Parque Estadual de Vila Velha, no Segundo Planalto Paranaense. A partir de novembro de 2004, foi 
encontrada na localidade Juruqui (Chácara Sandri), em Almirante Tamandaré. O adulto tem coloração negra metálica e cerca de $5 \mathrm{~mm}$ de comprimento. Cabeça, pronoto e abdome são claramente distintos. A pontuação de seus élitros é paralela e visível sob o microscópio-estereoscópio. É lento em temperaturas abaixo de $15{ }^{\circ} \mathrm{C}$, mas bastante ágil em temperaturas acima de $22{ }^{\circ} \mathrm{C}$. O macho é menor do que a fêmea. Seu vôo também se assemelha ao vôo de $B$. cinctipenis. Seu acasalamento foi observado no campo e no laboratório. A postura ocorre sobre folhas e ramos de $T$. fluminensis. O ovo tem a forma de bastonete e é de cor negra. A larva tem mancha amarela e parda, atinge até $6 \mathrm{~mm}$ de comprimento e tem forma do tipo "gota". Suas seis falsas pernas situam-se a $1 / 3$ da parte dianteira do corpo (pronoto). A larva é voraz e alimenta-se da parte abaxial da folha, sem danificar a película adaxial. Tem o hábito de transportar seus próprios excrementos na parte dorsal do corpo, provavelmente como um elemento de defesa. O casulo é de coloração leitosa, a forma é ovalada e repleto de acúleos. O empupamento ocorre em três semanas. Testes preliminares, em placa de Petri, indicaram sua preferência por T. fluminensis. Não ataca Capsicum sp., Lycopersicum esculentum Mill (Solanaceae), Phaseolus vulgaris L., Solanum gilo Raddi, Solanum melongena L., Spinacia oleracea L.

Tabela 11.Teste de preferência alimentar de crioceríneo (Coleoptera: Chrysomelidae) com chance e sem chance de escolha.

Table 11. Feeding preference test of Criocerinae (Coleoptera: Chrysomelidae) with choice and no choice.

\begin{tabular}{lcc|cccc}
\hline \multicolumn{3}{c}{ Planta hospedeira } & \multicolumn{4}{c}{ Ambiente de teste } \\
\hline \multirow{2}{*}{$\begin{array}{l}\text { Nome } \\
\text { comum }\end{array}$} & $\begin{array}{c}\text { Nome } \\
\text { científico }\end{array}$ & \multirow{2}{*}{ Família } & \multicolumn{3}{c}{ Petri } & \multicolumn{3}{c}{ Gaiola } \\
\cline { 3 - 7 } & & Sem chance & $\begin{array}{c}\text { Com chance } \\
\text { de escolha }\end{array}$ & $\begin{array}{c}\text { Sem chance } \\
\text { de escolha }\end{array}$ & $\begin{array}{c}\text { Com chance } \\
\text { de escolha }\end{array}$ & escolha \\
\hline Agrião & N. officinalle & Brassicae & 0 & 0 & NT & NT \\
Alface & L. sativa & Asteraceae & 0 & 0 & NT & NT \\
Espinafre & S. oleracea & Chenopodiaceae & 0 & 0 & NT & NT \\
T. zebrina & T. zebrina & Commelinaceae & 0 & 0 & NT & NT \\
Beringela & S. melongena & Solanaceae & 0 & 0 & NT & NT \\
Feijão & P. vulgaris & Fabaceae & 0 & 0 & NT & NT \\
Jiló & S. gilo & Solanaceae & 0 & 0 & NT & NT \\
Pimenta & Capsicum sp. & Solanaceae & 0 & 0 & NT & NT \\
Tomate & L. esculentum & Solanaceae & 0 & 0 & NT & NT \\
\hline
\end{tabular}

0: não danificado (no damaged); NT: não testado (no tested).

O besouro crioceríneo, comparado com a espécie $B$. cinctipennis, tem maior potencial como candidato ao controle biológico de $T$. fluminensis, pois pode ter várias gerações por ano e causa consideráveis danos às folhas, cujo impacto na planta é maior. Essa espécie está sendo submetida aos testes de interação inseto-planta, especificidade e de impacto. Entretanto, B. cinctipennis leva vantagem sobre o besouro crioceríneo porque suas larvas causam danos tanto nos ramos como nas folhas. Esse fator torna-se relevante, uma vez que a planta apresenta capacidade de se propagar através dos ramos e alta capacidade de enraizamento em ramos sem folhas. No entanto, o aspecto negativo observado é que $B$. cinctipennis reproduz-se com dificuldade em laboratório, e no campo foi observada apenas uma geração por ano. Em contrapartida, o besouro crioceríneo tem como principal vantagem sua facilidade de criação em laboratório. Outra vantagem do besouro crioceríneo é que, dentre as plantas testadas, mostrou especificidade a $T$. fluminensis (Tabela 11). A sua eficiência para o controle biológico deve ser comprovada em outros testes. Numa primeira análise, sugere-se a utilização conjunta dessas duas espécies e ainda a integração com outros insetos, como, por exemplo, Scirtothrips sp. Há necessidade do prosseguimento dos estudos e pesquisas sobre esses e outros candidatos para o controle biológico de $T$. fluminensis.

\section{AGRADECIMENTOS}

Agradecimentos a "Landcare Research New Zealand Limited", pelo suporte financeiro, e à FUPEF do Paraná, pela administração dos recursos e das bolsas dos estagiários. Ao Centro de Estações Experimentais do Canguiri - Fazenda Canguiri, pela assistência prestada no campo. Ao Dr. Clark do Life 
Science Museum, Brigham Young University (Provo, Utah, USA), que identificou a espécie $B$. cinctipennis. Ao Instituto Ambiental do Paraná (IAP), por permitir visita ao Parque Estadual de Vila Velha, onde T. fluminensis e T. zebrina encontram-se como invasoras. Ao Dr. Robert Weingart Barreto, da Universidade Federal de Viçosa, e seus orientados, pela cooperação no abastecimento de mudas de $T$. fluminensis e de C. diffusa. À família Sandri e ao Clube Atlético Primavera, por permitirem coleta de material em suas propriedades. À Professora Dra. Cibele Stramare Ribeiro Costa, da Universidade Federal do Paraná, pela tentativa de identificar o besouro crioceríneo. À Dra. Renata Chiarini Monteiro, da Universidade de São Paulo (ESALQ), pela identificação do gênero Scirtothrips. À Dra. Augusta Karkow Doetzer, da Universidade Federal do Paraná, e ao Prof. Dr. Julio C. Medal, da Universidade da Flórida, pela revisão do manuscrito. Aos pesquisadores do Laboratório Neotropical de Controle Biológico de Plantas, pelo apoio físico e moral.

\section{REFERÊNCIAS}

BLOSSFELD, H. Jardinagem. São Paulo: Biblioteca Agronômica Melhoramentos. 1965. p. 45, 93, 114, 124.

CARPANEZZI A. A.; FERREIRA, C. A.; ROTTA, E.; NAMIKAWA, I. S.; STURION, J. A.; PEREIRA, J. C. D.; MONTAGNER, L. H.; RAVEN, M. J.; CARVALHO, P. E. R.; SILVEIRA, R. A.; ALVES, S. T. Zoneamento ecológico para plantios florestais no Estado do Paraná. Brasília. EMBRAPA-CNPF, Documentos, n. 17, p. 1-89, 1986. LANGELAND, K. A.; BURKS, K. C. Tradescantia fluminensis Vell. (Commelinaceae) Dayflower Family - Green Wandering Jew. In: Identification \& Biology of Non-native Plants in Florida's Natural Areas. Gainesville: University of Florida. 1998. p.26-27.

MENDONÇA, R. C. DE; FELFILI, J. M.; WALTER, B. M. T.; SILVA-JR, M. C. DA; REZENDE, A. V.; FILGUEIRAS, T. S.; NOGUEIRA, P. E. Flora Vascular do Cerrado. In: SANO, S. M.; ALMEIDA, S. P. DE (Ed.). Cerrado: ambiente e flora. Planaltina DF: EMBRAPA - CPAC,. 1998. p.289.

ROY, B.; POPAY, I.; CHAMPION, P.; JAMES, T.; RAHMAN, A. An Illustrated Guide to Common Weeds of New Zealand. Hastings: N.Z. Plant Protection Society, 1998. p. 126.

TAKedA, I. J. M.; FARAGO, P. V. Vegetação do Parque Estadual de Vila Velha: guia de campo. Curitiba: Botânica, 2001. v.1. p. 277.

TOFT, R. J.; HARRIS, R. J.; WILLIAMS, P. A. Impacts of the weed Tradescantia fluminensis on insect communities in fragmented forests in New Zealand. Biological Conservation, Liverpool, n. 102, p. 3146, 2001. 